\title{
A Hybrid Modified PSO System Identification Method Based on the Asynchronous Time-Dependent Learning Factor
}

\author{
Jiangtao Zhai ${ }^{1}$, Chengming Zhu ${ }^{2}$, Chi He ${ }^{3 *}$, Zhijun Yao ${ }^{1}$ and Yuewei Dai ${ }^{1}$ \\ ${ }^{1}$ School of Automation, Nanjing University of Science and Technology, 200 Xiaolingwei Street, Nanjing, China \\ ${ }^{2}$ School of Electronic Information, Jiangsu University of Science and Technology, 2 Mengxi Road, Zhenjiang, China \\ ${ }^{3}$ School of Mechatronic Engineering, CUST, 7089 Weixing Road, Changchun, China \\ ${ }^{*}$ Corresponding author
}

\begin{abstract}
In this paper, the system identification method to Hammerstein model is studied. Considered that the identification accuracy of the standard particle swarm optimization (PSO) is limited and the local optimal problem is easily occurred at later stage, the standard PSO and its initial value setting is firstly discussed. Then, a modified PSO combined with the methods of asynchronous time-varying learning factor and linearly decreasing time-varying weight is put forward to obtain the optimal solution in the whole parameter space. Finally, the comparison experiments are done to verify the accuracy and the advantage of noise resistance of the proposed method.
\end{abstract}

Keywords-component; system identification; hammerstein model; particle swarm optimization; simulation

\section{INTRODUCTION}

Now the algorithms of system identification mainly includes least squares algorithm, neural network algorithm and swarm intelligence algorithm. These algorithms have been studied a lot, for example, recursive least squares algorithm was used to identify the model parameters ${ }^{[1]}$ and neural networks was also used to achieve an effective identification ${ }^{[2]}$. Swarm intelligence algorithm specifically includes PSO and the fruit flies algorithm i.e. PSO is a kind of random optimization algorithm based on group behaviors. It has the advantages of simple implementation and less adjustable parameters, which attracts the attention of many researchers. In reference[3], PSO is used for system identification, and in reference $[4,5]$, it is modified to identify some specific systems.

The inertia weight w, convergence factor (constraint factor), "cognitive"part c1 and "social" part c2 are the main factors which will influence the performance of PSO. A satisfying recognition effect can be obtained by adjusting and improving these main factors. Many improved methods have been proposed in the field of recognition at home and abroad. The dynamic inertia weight $w$ can achieve better search results than a fixed value. In the later stage, the value of $\mathrm{w}$ should be decreased to increase the search accuracy. Nevertheless, it still remains two points of weakness. One is that in the early stage, the local search ability is weak, and in the later stage, the global search ability is weak, which will easily bring local extreme. The other is the number of iterations is hard to predict, which will affect the regulating function of the algorithm. Three kinds of nonlinear decreasing weight strategy were proposed on the base of LDW ${ }^{[6]}$. The strategy of LDW is better than the convex function decreasing, but worse than the concave function decreasing. Yet the difference of the effect among these three strategies is not very obvious. An optimized PSO, i.e. PSO-DT, was attached with an optimal perturbation term through the velocity update mechanism of PSO ${ }^{[7]}$. This improvement could avoid the particles trapped in local optimum. However, the identification accuracy of PSO-DT is weak, that's because the introduction of the optimal perturbation terms is equivalent to bring in some noise interference, which may have certain influence on the identification accuracy.

Thus, a modified method based on the standard PSO is proposed in this paper. Specifically, the learning ability coefficient $\mathrm{c} 1, \mathrm{c} 2$ and inertia weight $\mathrm{w}$ of standard PSO is combined and adjusted through dynamic self-adapting to increase the search speed of particle and the ability of identification accuracy. Experimental comparison is carried out between the standard PSO and the modified PSO, which proves that the algorithm proposed in this paper can not only improve the identification accuracy, but also increase the convergence speed, and effectively avoid the local optimum as well.

\section{PARAMETER IDENTIFICATION OF THE HAMMERSTEIN MODEL BASED ON THE MODIFIED PSO}

\section{A. The Basic Introduction to PSO}

Given the ith particle position is $\mathrm{x}(\mathrm{i})=\left[\mathrm{x}_{\mathrm{i} 1}, \mathrm{x}_{\mathrm{i} 2}, \ldots, \mathrm{x}_{\mathrm{in}}\right]^{\mathrm{T}}$, and its velocity is $\mathrm{v}_{\mathrm{i}}=\left[\mathrm{v}_{\mathrm{i} 1}, \mathrm{v}_{\mathrm{i} 2}, \ldots, \mathrm{v}_{\text {in }}\right]^{\mathrm{T}}$. The individual extreme that each particle searched so far is $\mathrm{p}_{\mathrm{i}}=\left[\mathrm{p}_{\mathrm{i} 1}, \mathrm{p}_{\mathrm{i} 2}, \ldots, \mathrm{p}_{\mathrm{in}}\right]^{\mathrm{T}}$, which is indicated by pbest, and the extreme value of populations searched so far is $p_{g}=\left[p_{g 1}, p_{g 2}, \ldots, p_{g n}\right]^{T}$, indicated by $G_{\text {best. }}$ By applying the principle of tracking the currently known optimal location, the location velocity is updated by (1).

$$
\left\{\begin{array}{l}
v_{i d}(t+1)=w(t) v_{i d}(t)+\varphi \\
\varphi=c_{1} r_{1}\left[p_{i d}(t)-x_{i d}(t)\right]+c_{2} r_{2}\left[p_{g d}(t)-x_{i d}(t)\right] \\
x_{i d}(t+1)=x_{i d}(t)+v_{i d}(t+1)
\end{array}\right.
$$

where $\mathrm{d}=1,2, \ldots, \mathrm{n}, \mathrm{n}$ is the dimension index of the solution 
space, that is as to say the number of unknown parameters. $i=1$, $2, \ldots, \mathrm{m}, \mathrm{m}$ is the size of the population, $\mathrm{t}$ is the current times of iteration. $\mathrm{r} 1$ and $\mathrm{r} 2$ are uniformly distributed random numbers in the interval $[0,1] . \mathrm{c} 1$ and $\mathrm{c} 2$ are accelerating factors, also called learning coefficients, which presents the cognitive for its own and its entire group knowledge. $\mathrm{w}$ is called inertia weight, which is used to maintain the original velocity factor and balance the global and local convergence.

\section{B. The Modified PSO}

The main idea of the modified PSO proposed by this paper is that inertia weight $\mathrm{w}$, "cognitive" section $\mathrm{cl}$, and "social" part c2 are adjusted dynamically. In order to archive better performance, the inertia weight of the algorithm needs gradual linear decrease as the iteration proceeds. In the early stage of iteration, larger inertia weight can make the algorithm not easy to fall into a local optimum, and in the later stage, smaller one can make the optimization process converge faster and more smoothly to ensure a stable system convergence. The specific expression is shown as (2). Where $\mathrm{w}_{\max }$ is the maximum inertia weight, wmin is the minimum inertia weight, $t$ is the current iteration times, iter is the total times of iteration.

$$
w=w_{\max }-t \frac{w_{\max }-w_{\min }}{i t e r}
$$

Asynchronous time-varying procedure can be described as follows: $\mathrm{c} 1$ is assigned to be a larger value at the beginning of the particle swarm iteration and needs reduced gradually as the procedure proceeds. Instead, c2 starts with a smaller value and gradually increases itself later. The procedure is similar to the scenario that a group of people look for something: In the early stage, we rely on our own basic experience, just like the "selfcognitive" part c1. With the experience accumulated and consensus collected, we began to apply the social knowledge in looking for something, just like the "social knowledge" part c2. The relationship between $\mathrm{c} 1, \mathrm{c} 2$ and the times of iteration is shown in (3).

$$
\left\{\begin{array}{l}
c_{1}=\left(c_{1 f}-c_{1 i}\right) \times \frac{\text { iter }_{\text {max }}-t}{\text { iter }_{\text {max }}+t}+c_{1 i} \\
c_{2}=\left(c_{2 f}-c_{2 i}\right) \times \frac{i \text { iter }_{\text {max }}-t}{\text { iter }_{\text {max }}+t}+c_{2 i}
\end{array}\right.
$$

where itermax is the maximum number of particle swarm iterations, and $t$ is the current number of iterations, $c_{1 f}, c_{1 i}, c_{2 f}$, $\mathrm{c}_{2 \mathrm{i}}$ are constant.

\section{Parameter Identification of the Hammerstein Model Based on Modified PSO}

The identification of Hammerstein model is to estimate a set of optimized parameter vector $\theta$, which produces the minimum sum of squared errors between the parameter model output and the actual model output under a specified target function criteria. The identification parameter vector is set as: $\theta=\left(r_{2}, r_{3}, \cdots, r_{p}, a_{1}, a_{2}, \cdots, a_{m}, b_{1}, b_{2}, \cdots, b_{n}\right)$, and the parameter estimation vector is set as : $\vec{\theta}=\left(\vec{r}_{2}, \vec{r}_{3}, \cdots, \vec{r}_{p}, \vec{a}_{1}, \vec{a}_{2}, \cdots, \vec{a}_{m}, \vec{b}_{1}, \vec{b}_{2}, \cdots, \vec{b}_{n}\right)$.The purpose of the identification is to estimate a vector $\vec{\theta}$ which can satisfy the objective function. The objective function is the sum of squared errors between parameter model output and the actual model output. It can be described as (4):

$$
f(\theta)=\sum_{i=1}^{s}[y(k-i)-\bar{y}(k-i)]^{2}
$$

where $\mathrm{s}$ is the identification window size, $\bar{y}$ is the estimated output of the parameter model. The smaller value of the objective function achieves the better performance.The steps of the identification are as follows:

Step1: Initialize the population, including the population size and the dimensions of particles, as well as the maximum times of iteration, and assign the initial loop variable $\mathrm{t}=1, \mathrm{w}$, $\mathrm{c} 1$ and $\mathrm{c} 2$;

Step2: Generate the initial parameter space of identification by randomly assigning initial position, initial velocity, and individual extremum $P_{\text {best }}$ of each particle and global extremum gbest of the particle swarm;

Step3: Recalculate the position and velocity of every particle by applying (2-4) and then the parameter space of identification is updated;

Step4: Calculate the new fitness value of each particle through the target function;

Step5: For each particle, the new calculated fitness value is set as its individual extremum if the fitness value is less than the current individual extremum $P_{\text {best }}$; Find the minimum extremum among the particle swarm, set the minimum extremum as the current global extremum $G_{b e s t}$ if the minimum extremum is less than $G_{b e s t ;}$

Step6: If the current iteration times reaches preset maximum value, the loop terminates. Otherwise go to step 3. When the condition is met, the optimal parameter estimate and the optimal function resolution can be achieved.

\section{EXPERIMENTAL RESULTS AND ANALYSIS}

In order to verify the effectiveness of the proposed algorithm in, the model is selected as (5).

$$
\left\{\begin{array}{l}
A\left(q^{-1}\right) y(k)=B\left(q^{-1}\right) x(k)+\xi(k) \\
x(k)=u(k)+0.5 u^{2}(k)+0.3 u^{3}(k) \\
A\left(q^{-1}\right)=1+1.5 q^{-1}-0.7 q^{-2} \\
B\left(q^{-1}\right)=q^{-1}+0.5 q^{-2}
\end{array}\right.
$$

Where $\mathrm{u}(\mathrm{k})$ is a Gaussian White noise sequence and $\xi(\mathrm{k})$ is a noise sequence. The mean and variance of $u(k)$ are respectively 0 and 1 , while the mean and variance of $\xi(\mathrm{k})$ is 0 and 0.1 . There are 6 parameters in (5) needed to be identified, which are shown in (6): 


$$
\theta=\left(r_{2}, r_{3}, a_{1}, a_{2}, b_{1}, b_{2}\right)
$$

The parameters of PSO are set as follows: the window size $\mathrm{s}$ is 50 , the number of particle population $\mathrm{n}$ is 40 , and the maximum number of iterations iter is 1200 . The maximum velocity $\mathrm{v}_{\max }$ is 1 and the maximum location $\mathrm{x}_{\max }$ is 2 . The optimal parameter estimation of this model is conducted using modified PSO and standard PSO respectively, and the comparison results are shown in Figure I.The output curve of the model proposed in this paper is in accordance with that of the original model. It shows an excellent identification effect and verifies the feasibility and effectiveness of the proposed modified PSO, which is combined with asynchronous timevarying linear learning factor method and linear time-varying weight method. Another thirty times experiments have been done to illustrate the effectiveness of the proposed method, and the experimental results are shown in Table I.

In Table I, the identification precision of the improved PSO is higher than that of the standard PSO, thus verifying that the modified PSO algorithm has the ability to improve the precision of model identification. The changing process of identification parameters is shown in Figure II and III.

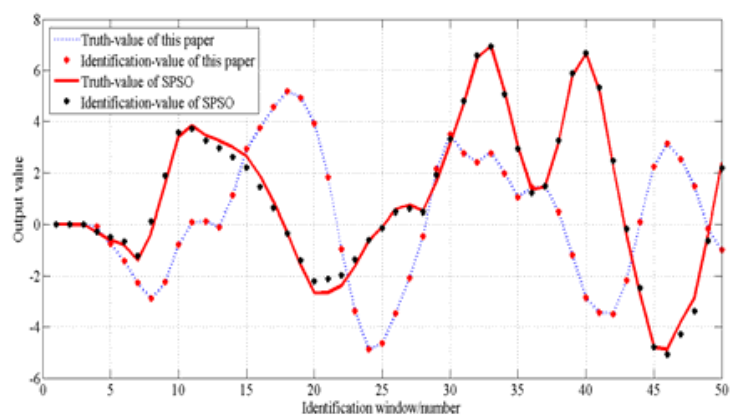

FIGURE I. THE COMPARISON OF IDENTIFICATION EFFECT BETWEEN MODIFIED PSO AND STANDARD PSO

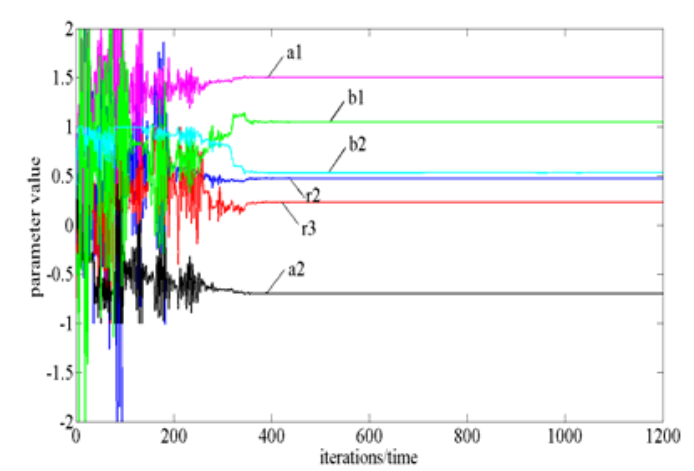

FIGURE II. THE CHANGING PROCESS OF IDENTIFICATION PARAMETERS OF THE MODIFIED PSO

From Figure II and III, it can be seen that the identification parameters of the modified PSO match the truth values of the real model when the number of iterations reaches 400 approximately, while the standard PSO algorithm cannot match the truth values until the number of iterations reaches about 600 . In order to further illustrate the advantages of the proposed algorithm, the comparison is conducted in this papTer with two existing improved PSO algorithms. One is carried with the constriction factor, and the other is carried with the perturbation factor. A brief introduction of the Contraction Factor Particle Swarm Optimization (CFPSO): The constriction factor $\lambda$ is introduced to keep the convergence of PSO. It conducts the search by changing the speed updating formula of the particle while keeping the location updating formula unchanged. The speed updating formula is shown in (7).

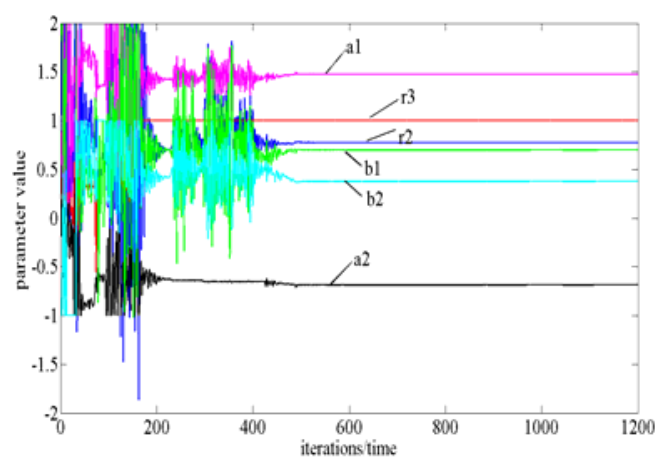

FIGURE III. THE CHANGING PROCESS OF IDENTIFICATION PARAMETERS OF THE STANDARD PSO

TABLE I. THE PARAMETERS AND IDENTIFICTION EFFECTS OF THE MODIFIED PSO AND STANDARD PSO

\begin{tabular}{|c|c|c|c|c|c|}
\hline \multirow{2}{*}{$\begin{array}{c}\text { Vari } \\
\text { able }\end{array}$} & \multirow{2}{*}{$\begin{array}{c}\text { Truth } \\
\text { Value }\end{array}$} & \multicolumn{2}{|c|}{ Present algorithm } & \multicolumn{2}{|c|}{ SPSO } \\
\cline { 3 - 6 } & & Mean & $\begin{array}{c}\text { Standard } \\
\text { deviation }\end{array}$ & Mean & $\begin{array}{c}\text { Standard } \\
\text { deviation }\end{array}$ \\
\hline$r_{2}$ & 0.5 & 0.4969 & 0.0067 & 0.4948 & 0.0194 \\
\hline$r_{3}$ & 0.3 & 0.2925 & 0.0253 & 0.2789 & 0.0794 \\
\hline$a_{1}$ & 1.5 & 1.4994 & 0.0026 & 1.4953 & 0.0170 \\
\hline$a_{2}$ & -0.7 & -0.6993 & 0.0023 & -0.6965 & 0.0125 \\
\hline$b_{1}$ & 1 & 1.0046 & 0.0167 & 0.9871 & 0.0416 \\
\hline$b_{2}$ & 0.5 & 0.5033 & 0.0101 & 0.5472 & 0.1539 \\
\hline
\end{tabular}

TABLE II. THE PARAMETERS AND DDENTIFICTION EFFECTS OF THE MODIFIED PSO AND STANDARD PSO

\begin{tabular}{|c|c|c|c|c|c|}
\hline \multirow{2}{*}{$\begin{array}{c}\text { Varia } \\
\text { ble }\end{array}$} & \multirow{2}{*}{$\begin{array}{c}\text { Truth } \\
\text { value }\end{array}$} & \multicolumn{2}{|c|}{ Present algorithm } & \multicolumn{2}{c|}{ CFPSO } \\
\cline { 3 - 6 } & & $\begin{array}{c}\text { Standard } \\
\text { deviation }\end{array}$ & Mean & $\begin{array}{c}\text { Standard } \\
\text { deviation }\end{array}$ \\
\hline$r_{2}$ & 0.5 & 0.4969 & 0.0067 & 0.4952 & 0.0180 \\
\hline$r_{3}$ & 0.3 & 0.2925 & 0.0253 & 0.2832 & 0.0605 \\
\hline$a_{1}$ & 1.5 & 1.4994 & 0.0026 & 1.4970 & 0.0114 \\
\hline$a_{2}$ & -0.7 & -0.6993 & 0.0023 & -0.6978 & 0.0080 \\
\hline$b_{1}$ & 1 & 1.0046 & 0.0167 & 0.9998 & 0.0309 \\
\hline$b_{2}$ & 0.5 & 0.5033 & 0.0101 & 0.5246 & 0.1013 \\
\hline
\end{tabular}

$$
\left\{\begin{array}{l}
v_{i d}(t+1)=\lambda \cdot \varepsilon \\
\varepsilon=v_{i d}(t)+c_{1} r_{1}\left[p_{i d}(t)-x_{i d}(t)+c_{2} r_{2}\left[p_{g d}(t)-x_{i d}(t)\right]\right.
\end{array}\right.
$$

where $\lambda=2 /|2-\varphi-\sqrt{\varphi(\varphi-4)}|, \varphi=\mathrm{c} 1+\mathrm{c} 2, \varphi>4$. $\varphi$ is set to be 4.1 , and the constriction factor $\lambda$ is calculated to be 0.729 . The comparison of identification effect between the improved PSO with constriction factor and the proposed algorithm is presented in Figure IV. 


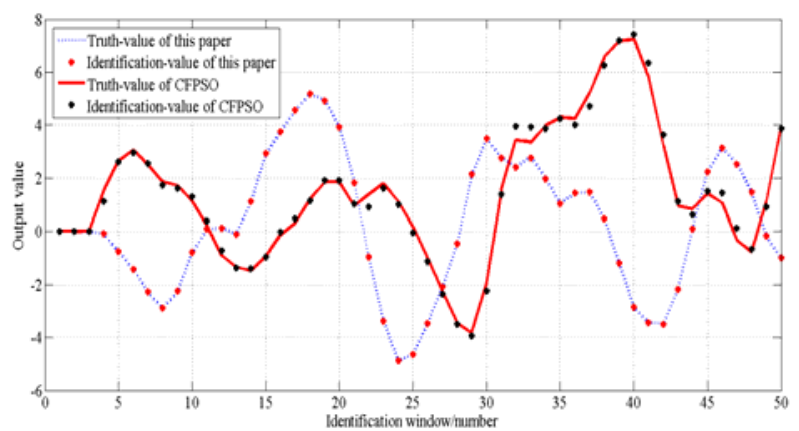

FIGURE IV. THE COMPARISON OF IDENTIFICATION EFFECT BETWEEN IMPROVED PSO WITH CONSTRICTION FACTOR AND THE PROPOSED ALGORITHM

In Figure IV, the curve of the identification value in the proposed algorithm can fit the curve of truth value very well, which means high identification precision. While the output curve of the PSO with constriction factor doesn't match the truth value well, which means its identification precision is not good enough. The comparison of identification parameters after 30 simulation experiments between the proposed algorithm and the improved PSO with constriction factor is presented in Table II.As seen from Table II, the mean values of the identification parameters of the proposed algorithm are closer to the true values than those of the improved PSO with constriction factor, which means better performance in precision and effect of identification and fulfills our expectations.

There exists an improved particle swarm optimization algorithm with disturbance term (PSO-DT), which added an optimal perturbation term to the speed update mechanism of PSO. Its purpose is to avoid particles trapped in local optimum. The specific formula is described as (8).

$$
\left\{\begin{array}{l}
v_{i d}(t+1)=w(t) v_{i d}(t)+c_{1} r_{1}\left[p_{i d}(t)-x_{i d}(t)\right]+\psi \\
\psi=c_{2} r_{2}\left[p_{g d}(t)-x_{i d}(t)\right]+a\left(r_{3}-0.5\right)
\end{array}\right.
$$

In (8), a is a small constant, $\mathrm{c} 1, \mathrm{c} 2$ are learning factors, $\mathrm{r} 1$, $\mathrm{r} 2, \mathrm{r} 3$ are random numbers in the range of $[0,1]$.

The comparison of identification parameters after 30 simulation experiments between the proposed algorithm and the PSO-DT is presented in Table III.

THE PARAMETERS AND IDENTIFICTION DFFECTS OF THE MODIFIED PSO AND STANDARD PSO

\begin{tabular}{|c|c|c|c|c|c|}
\hline \multirow{2}{*}{$\begin{array}{c}\text { Varia } \\
\text { ble }\end{array}$} & \multirow{2}{*}{$\begin{array}{c}\text { Trut } \\
\text { hvalu } \\
\mathbf{e}\end{array}$} & \multicolumn{2}{|c|}{ Present algorithm } & \multicolumn{2}{|c|}{ PSO-DT } \\
\cline { 3 - 6 } & Mean & $\begin{array}{c}\text { Standard } \\
\text { deviation }\end{array}$ & Mean & $\begin{array}{c}\text { Standard } \\
\text { deviation }\end{array}$ \\
\hline$r_{2}$ & 0.5 & 0.4969 & 0.0067 & 0.4951 & 0.0524 \\
\hline$r_{3}$ & 0.3 & 0.2925 & 0.0253 & 0.2579 & 0.2012 \\
\hline$a_{1}$ & 1.5 & 1.4994 & 0.0026 & 1.5047 & 0.0200 \\
\hline$a_{2}$ & -0.7 & -0.6993 & 0.0023 & -0.7074 & 0.0234 \\
\hline$b_{1}$ & 1 & 1.0046 & 0.0167 & 1.0265 & 0.1357 \\
\hline$b_{2}$ & 0.5 & 0.5033 & 0.0101 & 0.4979 & 0.1602 \\
\hline
\end{tabular}

Table III shows that the mean values of identification parameters of the proposed algorithm are closer to truth values than those of PSO-DT. Therefore, it is easily concluded that the identification precision of the proposed algorithm is better than PSO-DT.

\section{CONCLUSIONS AND FUTURE WORKS}

In this paper, the Hammerstein model of single input and single output is identified by the introduction of a modified PSO, which is combined with asynchronous time-varying linear learning factor method and linear time-varying weight method. From the experimental results, it can be seen that the modified PSO can not only increase the identification accuracy, but also improve the convergence speed and convergence effect. Also, it has also demonstrated the effectiveness of the algorithm by preventing the system from falling into local optimization and achieving ideal identification results.

However, it should be noticed that the robustness of the proposed algorithm is not strong enough under the circumstance of different signal-to-noise ratio, which needs to be further studied in the future.

\section{ACKNOWLEDGMENT}

This work is supported by the National Science Foundation (U1636117), National Science Foundation of Jiangsu Province (Grant No. BK20150472).

\section{REFERENCES}

[1] Ding, F., Wang, Y. J. and Ding, J., 2015. Recursive least squares parameter identification algorithms for systems with colored noise using the filtering technique and the auxiliary mode. Digital Signal Processing, (37), 100-108.

[2] Linhares, L., Fontes, A. and Martins, A. M., 2015. Fuzzy wavelet neural network using a correntropy criterion for nonlinear system identification. Mathematical Problems in Engineering, Article ID 678965, 1-12.

[3] Upadhyay, P., Kar, R. and Mandal, D., 2014. Craziness based particle swarm optimization algorithm for IIR system identification problem. International Journal of Electronicsand Communications (AEÜ), (68):369-378.

[4] Huang, Y., Xiao, T. T. and Han, P., 2014. An improved quantum particle swarm optimization and its application in system identification. In The 26th Chinese Control and Decision Conference (2014 CCDC), 11321134.

[5] Ma, R. Y., Yang, L. L. and Zhang, Z. C., 2013. Analysis the characteristic of $\mathrm{C} 1, \mathrm{C} 2$ based on the PSO of iterative shift and trajectory of particle. Mathematical Computation, 2(4), 109-115.

[6] Chen, G. M., Jia, J. Y. and Han, Q., 2006. Particle swarm optimization algorithm is used to decreasing inertia weight strategy research. Journal of Xi'an Jiaotong University, 40(1), 53-61.

[7] Li, X. F., Xue, B. J. and Li, W. Q., 2008. Based on improved particle swarm algorithm of reservoir optimal operation research. Water Power, 34(11), 107-109. 